\title{
Subcutaneous emphysema: a rare manifestation of a perforated diverticulitis in a patent inguinal canal
}

\author{
Lambert de Vries • Anne-Sophie Knoepfli • \\ Philippe Konstantinidis • Emmanuel Charbonney
}

Received: 29 August 2006 / Accepted: 9 November 2006 / Published online: 29 November 2006

(c) Springer-Verlag 2006

\begin{abstract}
Patients with complicated diverticulitis rarely present with extraperitoneal manifestations but the manifestation of subcutaneous emphysema appears even more seldom. We present the case of a patient with a history of diabetes and immunosuppression, who was admitted with sepsis in association with cellulitis and subcutaneous emphysema of the left groin. The absence of peritonism due to corticosteroid treatment, a history of a recent fall with an ilio- and ischiopubic fracture and subcutaneous emphysema led to a delay in the diagnosis. The final diagnosis was a perforated diverticulitis in a patent inguinal canal, which was only revealed after surgery. The various complications of diverticulitis, including extraperitoneal manifestations, and associated microorganisms implicated in cellulitis and subcutaneous emphysema are briefly reviewed.
\end{abstract}

Keywords Diverticulitis · Inguinal canal ·

Subcutaneous emphysema

L. de Vries

Internal Medicine Department, University Hospital Geneva, Rue Micheli-Du-Crest 24, 1211 Geneva 14, Switzerland

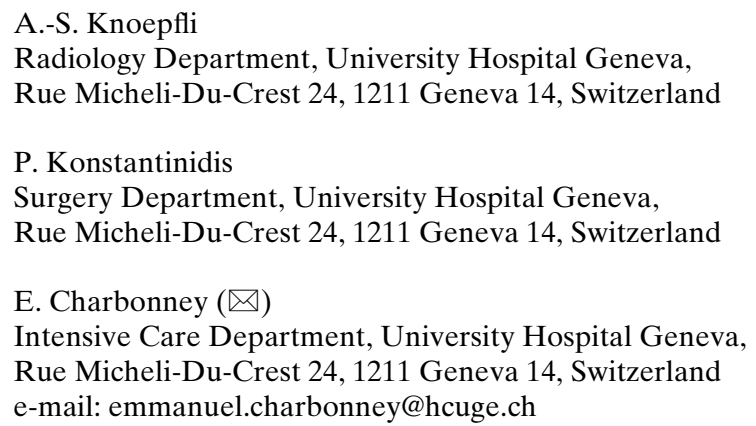

\section{Introduction}

One-third of patients with diverticulosis will develop diverticulitis. Intra-abdominal perforation, abscess, hemorrhage, intestinal stricture or obstruction and fistula are the most common complications. These patients frequently present with abdominal symptoms and, on occasion, with peritonism. However, in rare cases, complications outside of the peritoneal cavity have been reported [1].

\section{Case report}

An 82-year-old institutionalized female patient with a history of corticosteroid $(2 \times 40 \mathrm{mg} /$ day $)$ treatment for rheumatoid arthritis was admitted to our emergency department for dyspnea and left groin pain. These symptoms had appeared a week before admission, following a fall onto the left hip. Her medical history included corticosteroid-induced diabetes, arterial hypertension, ischemic cardiopathy and peritonitis.

Physical examination revealed a patient in a markedly diminished general condition, afebrile, tachypneic, tachycardic and hypotensive. The abdominal examination showed a previous laparotomy scar and predominant pain in the left lower quadrant on palpation, without signs of peritonism. A digital rectal examination could not be carried out due to pain.

The left groin showed an inflammatory swelling with crepitations upon palpation. The surrounding skin was ischemic with a beginning of central necrosis. Blood tests confirmed an inflammatory syndrome with high non-segmented neutrophiles and high C-reactive protein in addition to a discrete rhabdomyolysis, diabetes 
decompensation and acute renal failure. A pelvic Xray showed subcutaneous air and a left ilio- and ischiopubic fracture (Fig. 1).

Systemic inflammation response syndrome (SIRS) along with the physical examination and pelvic X-ray described above led to the following differential diagnoses: rupture of a hollow pelvic organ consecutive to pelvic fracture, cutaneous infection in relation to the fall (with no evidence of portal of entry) or an incarcerated inguinal hernia with secondary rupture.

In addition to initiating resuscitation and antibiotic treatment, we performed an unenhanced - due to acute renal failure - abdominal-pelvic computed tomography scan (CT) with contrast media in the bladder to investigate the possibility of a fistula or a ruptured bladder. The CT scan showed thickened intestinal loops in the lower left quadrant with a suspicion of communication with the subcutaneous air

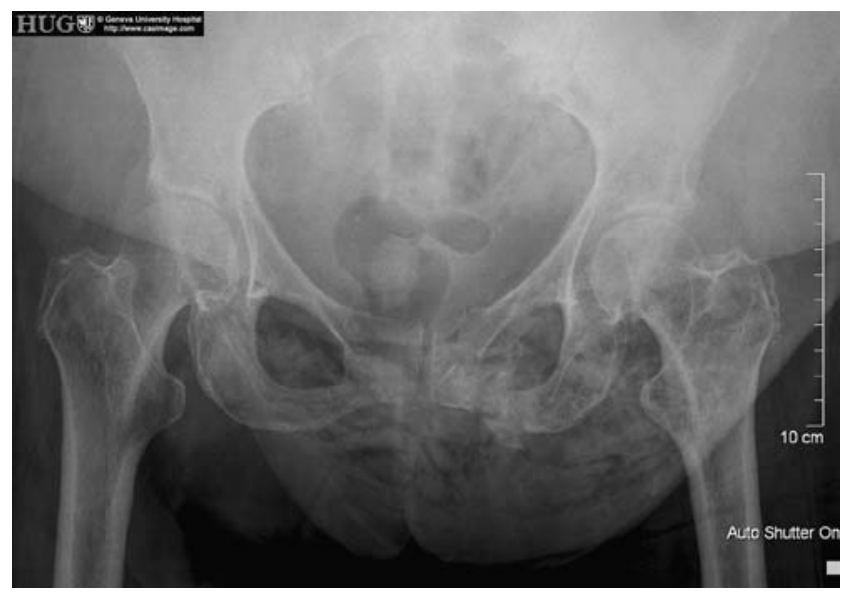

Fig. 1 Pelvic X-ray of the 82-year-old female patient showing subcutaneous air and a left ilio- and ischio-pubic fracture

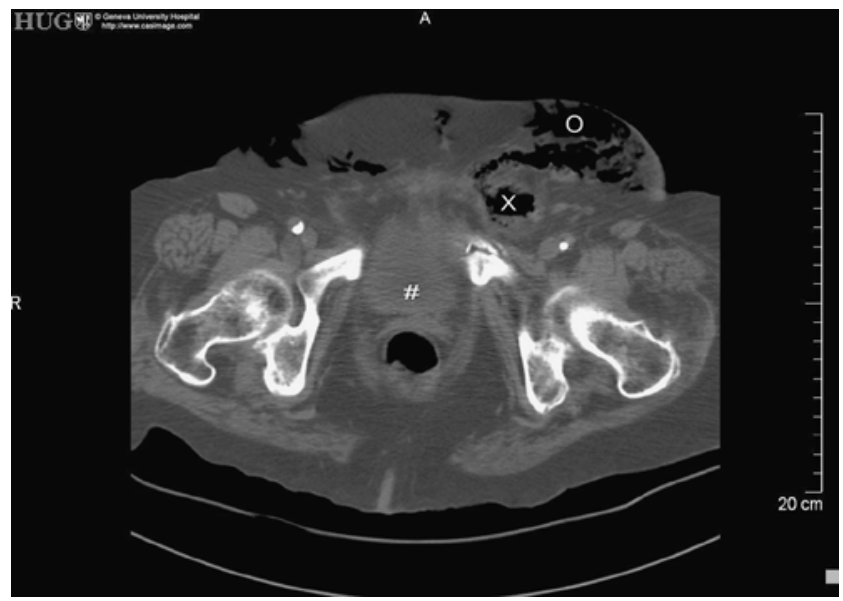

Fig. 2 Computed tomography scan of abdomen and pelvis of the 82-year-old patient. \# Foley catheter, $\mathrm{X}$ air containing collection, O subcutaneous emphysema associated with infiltration of the surrounding fat (Fig. 2). Radiological findings were consistent with an old left minimally displaced ilio- and ischio-pubic fractures due to the presence of a callus. There was neither a bladder breach nor an intestinal lesion; there was also no free air or free fluid in the peritoneal cavity.

An emergency surgical exploration of the groin region was decided upon. This showed cutaneous necrosis with an underlying abscess in an opening (containing no intestinal loop) leading to the peritoneal cavity. That opening was lateral, above the inguinal ligament through the direct floor. The laparotomy revealed a sigmoiditis, perforated along a length of $1 \mathrm{~cm}$, with an important inflammatory adhesion against the patent inguinal canal, which was observed from the outside; there was no other extension of the diverticulitis inside the peritoneal cavity. An Hartmann's sigmoidal resection with terminal colostomie was carried out. The hernia defect was closed with five separated stitches of 2.0 thread. Skin and tissue necrosis was extensively resected, the skin was closed with four loose separated stitches and a drain was left in place.

The surgical resection specimen confirmed diverticulosis associated with a perforated diverticular abscess. The final diagnosis was a perforated diverticulitis in a patent inguinal canal leading to subcutaneous abscedation and gas gangrene. In the postoperative period, our patient developed a progressive necrosis of the abdominal wall with septic shock and multiple organ failure which unfortunately led to her death.

\section{Discussion}

To our knowledge, this particular type of presentation of a perforated diverticulitis has never been described. The most common intra-peritoneal complications of diverticulitis are abscess formation $(46 \%)$, frank perforation $(21 \%)$, diverticular hemorrhage $(13 \%)$, intestinal stricture and occlusion (10\%) and fistula (10\%) [2]. Among the latter, colo-vesical fistulas are the most frequently encountered, followed by colo-vaginal, colouterine and finally colo-cutaneous fistula $(1-2 \%$ of all fistula cases) in decreasing order of prevalence.

Fistulas occur in more than $90 \%$ of cases after resection surgery for acute diverticulitis [3] or after percutaneous catheter drainage of an intra-abdominal abscess. Only a minority of the fistulas occur spontaneously, without any abdominal pathology, with corticosteroid treatment being a risk factor [4]. However extraperitoneal manifestations of diverticulitis are rare and result from fistulizations outside of the peritoneal cavity. Most reported cases are retroperitoneal fistulas 
presenting as thigh cellulites [5] that can also extend to the mediastinum and present thoracic or cervical subcutaneous emphysema [6]. Very few cases have been reported of a communication with mesenteric or portal veins involving the liver [7], the area of the appendix or epidural space. Subcutaneous emphysema can also result from a remote infection, and some dermatological manifestations are also described in the case of diverticulitis [8]. Our patient, who was on corticosteroid treatment for rheumatic arthritis [9], presented a clinically silent diverticulitis with this rare extra-peritoneal manifestation. The lower resistance of the patent's inguinal canal possibly allowed a perforation of the diverticular inflammation at this location.

Following pelvic trauma, one should suspect cutaneous infection secondary to local bacterial contamination. Once these microorganisms, typically Clostridium perfringens, have passed the skin barrier and are in anaerobic conditions [10], they can develop and produce toxins. The incubation period ranges from several hours to a few days. Cellulitis or necrosis then progresses to form an abscess, which ultimately develops into a severe systemic disease. Apart from Clostridium perfringens, Streptococcus group A or group B and Staphylococcus are frequently found in soft tissue infections, the former commonly encountered in diabetic patients. However, Enterobacteria, Bacteroides fragilis and Enterococcus, which are most commonly found in the peritoneal cavity, can also be involved. Nevertheless, gas gangrene essentially occurs as a result of infection by Clostridium species or other fermentation bacteria able to produce gas. No cutaneous portal of entry was found in our patient. The groin abscess was positive for Bacteroides fragilis and Clostridium perfringens, and the blood cultures were positive for Escherichia coli and Enterobacter, all of which are intestinal microorganisms.

\section{Conclusion}

Extra-abdominal rupture or fistulas are uncommon and atypical presentations of diverticulitis. Our patient showed very few specific symptoms in relation to the important immunosuppression by corticosteroids, making the diagnosis all the more difficult.

Immunosuppressed patients presenting with minimal digestive symptoms should receivecomplete radiological examinations to exclude the most probable diagnoses and extend potential diagnoses to the more unexpected possibilities. However, in the present case, the final diagnosis was only revealed after surgery.

\section{References}

1. Rothenbuehler JM, Oertli D, Harder F (1993) Extraperitoneal manifestation of perforated diverticulitis. Dig Dis Sci 38:1985-1988

2. McConnell EJ, Tessier DJ, Wolff BG (2003) Populationbased incidence of complicated diverticular disease of the sigmoid colon based on gender and age. Dis Colon Rectum 46:1110-1114

3. Fazio VW, Church JM, Jagelman DG et al. (1987) Colocutaneous fistulas complicating diverticulitis. Dis Colon Rectum 30:89-94

4. Finkelstein JA, Jamieson CG (1987) An association between anti-inflammatory medication and internal pelvic fistulas. Dis Colon Rectum 30:168-170

5. Edwards JD, Eckhauser FE (1986) Retroperitoneal perforation of the appendix presenting as subcutaneous emphysema of the thigh. Dis Colon Rectum 29:456-458

6. Cifuentes Tebar J, Aguayo Albasini JL, Robles Campos R et al. (1990) Subcutaneous emphysema as the initial manifestation of perforation of a hollow abdominal viscus. Rev Esp Enferm Dig 78:38-40

7. Sonnenshein MA, Cone LA, Alexander RM (1986) Diverticulitis with colovenous fistula and portal venous gas. Report of two cases. J Clin Gastroenterol 8:195-198

8. Kurgansky D, Foxwell MM JR (1993) Pyoderma gangrenosum as a cutaneous manifestation of diverticular disease. South Med J 86:581-584

9. Mpofu S, Mpofu CM, Hutchinson D et al. (2004) Steroids, non-steroidal anti-inflammatory drugs, and sigmoid diverticular abscess perforation in rheumatic conditions. Ann Rheum Dis 63:588-590

10. Mourvillier B, Bedos JP (2001) Soft tissue infections by anaerobic bacteria. Etiology, diagnosis, treatment. Rev Prat 51:319-324 